\title{
INFAMY: An Infinite-State Markov Model Checker ${ }^{\star}$
}

\author{
Ernst Moritz Hahn, Holger Hermanns, Björn Wachter, and Lijun Zhang \\ Universität des Saarlandes, Saarbrücken, Germany \\ \{emh, hermanns, bwachter, zhang\}@cs.uni-sb.de
}

\begin{abstract}
The design of complex concurrent systems often involves intricate performance and dependability considerations. Continuous-time Markov chains (CTMCs) are a widely used modeling formalism, where performance and dependability properties are analyzable by model checking. We present INFAMY, a model checker for arbitrarily structured infinite-state CTMCs. It checks probabilistic timing properties expressible in continuous stochastic logic (CSL). Conventional model checkers explore the given model exhaustively, which is often costly, due to state explosion, and impossible if the model is infinite. INFAMY only explores the model up to a finite depth, with the depth bound being computed on-the-fly. The computation of depth bounds is configurable to adapt to the characteristics of different classes of models.
\end{abstract}

\section{Introducing INFAMY}

Continuous-time Markov chains (CTMCs) are widely used in performance and dependability analysis and biological modeling. Properties are typically specified in continuous stochastic logic (CSL) [1], a logic inspired by CTL. In CSL, the until operator is equipped with a time interval to express properties such as: "The probability to reach a goal within 2 hours while maintaining a probability of at least 0.5 of communicating periodically (every five minutes) with a base station, is at least 0.9" via $\mathcal{P}_{\geq 0.9}\left(\left(\mathcal{P}_{\geq 0.5} \diamond^{\leq 5}\right.\right.$ communicate $) \mathcal{U} \leq 120$ goal $)$. CSL model checking amounts to analysis of the transient (time-dependent) probability vectors 11, typically carried out by uniformization, where the transient probability is expressed by a weighted infinite sum (weights are given by a Poisson process). The standard methodology in CSL model checking is to truncate the infinite sum up to some pre-specified accuracy [2]. Outside the model checking arena, ideas have been developed [345] which not only truncate the infinite sum, but also the matrix representing the system, which admits transient analysis of CTMCs with large or even infinite state spaces, provided they are given implicitly in a

\footnotetext{
* This work is supported by the NWO-DFG bilateral project VOSS, by the DFG as part of the Transregional Collaborative Research Center SFB/TR 14 AVACS and the Graduiertenkolleg "Leistungsgarantien für Rechnersysteme", and has received funding from the European Community's Seventh Framework Programme under grant agreement $\mathrm{n}^{\circ} 214755$.
}

A. Bouajjani and O. Maler (Eds.): CAV 2009, LNCS 5643, pp. 641-647, 2009.

(C) Springer-Verlag Berlin Heidelberg 2009 
modeling language. Harvesting and improving on these ideas, INFAMY is the first CSL model checker based on truncation. The underlying truncation technique was developed in [6]. Besides truncation, INFAMY features SPIN-like [7] state space exploration, and supports models given in a high-level description language.

Several other CSL model checkers exist, see 8 for an overview. Among them, PRISM [9] is a probabilistic model checker which uses advanced techniques for model representation and model checking for several stochastic model types. The model description language of INFAMY is based on the one of PRISM, but allows for infinite variable domains, while PRISM is restricted to finite models. Thus the tools are incomparable for infinite models. For several very large finite models, INFAMY is competitive with PRISM, as evident from Section 3. Model checkers based on discrete-event simulation [10/111219 could, in principle, also analyze models with implicitly infinite state space, however they have not yet been applied to such models, and thus we cannot compare with them.

INFAMY is available at http://depend.cs.uni-sb.de/ emh/infamy.

\section{Truncation-Based Model Checking}

INFAMY reads models in a guarded-command language extending the one of PRISM. The semantics of a model is a CTMC in which each state is a valuation of model variables. Initial states are specified by an expressions over model variables. The rest of the description consists of commands. Each command comprises a guard and a set of rates. Each rate is associated with an update formula. If a state fulfills the guard of a command, this state has a rate to each state obtained by the respective update formula. Contrary to PRISM, we allow variables with infinite range. Properties are specified in the time-dependent fragment of CSL which involves the Boolean operators, timed until and next operators.

Using truncation, we compute a finite submodel which is sufficient for checking the validity of a property given in time-dependent CSL. This is done by descending into the CSL formula and at the same time exploring the model subject to the different time bounds given in the subformulas as well as the rates occurring in the model (details can be found in [13). Beginning with a set of start states - either the initial states or, when descending into nested formulas, states determined by a subformula, the model is explored breadth-first up to

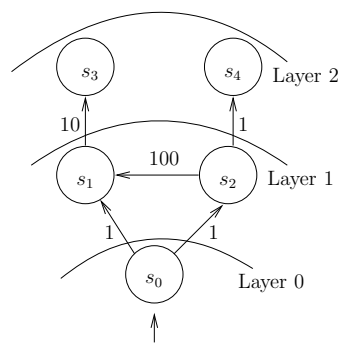

Fig. 1. Layers. a certain depth. The lower the depth the higher the error in the probabilities computed during model checking. This error needs to be kept below a userdefined absolute error $\varepsilon$ for all methods described in the following. As illustrated in Figure 1, our technique proceeds layer by layer exploring states with increasing depth, i.e. minimal distance from the start states, and estimates the error on the fly to determine if the depth is already sufficient. In the next paragraph, we discuss three error-estimation methods implemented in INFAMY, ranging from very precise and expensive to faster techniques with larger overestimation. An 
interesting trade-off arises between finding the smallest depth possible and the cost of error estimation.

Finite state projection (FSP). Munsky and Khammash [5] consider transient properties in the context of infinite-state biological models, including the one resulting from Chemical Master Equation problems. They build the CTMC incrementally in layers. Whenever adding a layer, they estimate the error by computing the probability of reaching the outermost layer from the initial states. FSP can find a minimal truncation point. However, as the exploration of new layers involves a stochastic analysis, relative computational cost of error estimation can be high.

Uniform. While FSP performs error estimation on the full model, we recently developed the Uniform method [13, which abstracts the model to a chain, as illustrated in Figure 3. The probability of reaching the last state of the chain is an upper bound for the error. Compared to FSP, this admits a much faster error estimation, where the chain needs not even be constructed explicitly. We can just consider the maximal sum of rates $\lambda_{\max }$ leaving a state into the next layer. Using the FoxGlynn algorithm [2], a right truncation point is

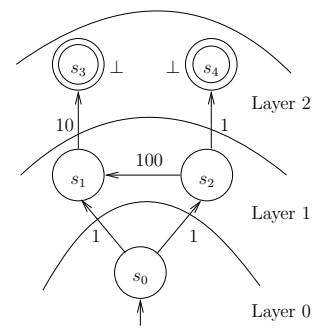

Fig. 2. FSP.

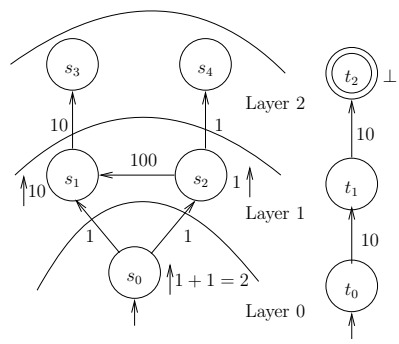

Fig. 3. Uniform method. computed by starting the algorithm with a value of $\lambda_{\max } \cdot t$ where $t$ is the timebound of the current subformula. Whenever a state with a higher sum is seen, we adjust the right truncation point.

Layered. We consider more fine-grained chains than in the Uniform method that take into account maximal rates for individual layers, leading to a chain in which each edge is labeled with the maximal rate of the corresponding layer. This is depicted in Figure 4. Using the vector, we can construct a birth process in which the probability of reaching the last state within time bound $t$ is larger than leaving the finite truncation. By building the birth process until we see a probability which is low enough, we obtain a method

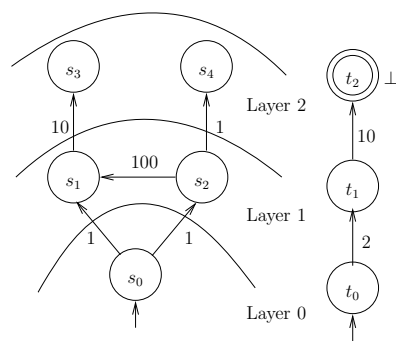

Fig. 4. Layered Method. which may explore less layers than the Uniform one if the rates of the layers vary. However, we have to compute the probabilities within the birth process each time we explore a new layer. Thus model exploration may be more timeconsuming. We call this the Layered method.

In all cases, after the exploration, usual CSL model checking is applied on the resulting submodel. 


\section{Results}

We compare different configurations of INFAMY, i.e., FSP, Uniform and Layered method. Further, for finite models, we assess the effectiveness of truncation by comparing with PRISM. We denote the number of states on which model checking is performed by $n$. For INFAMY, this refers to the finite truncation, and, for PRISM, this refers to the full model. Where appropriate, we denote multiples of 1000 by " $K$ ". For all configurations, the probabilities listed in the experiments are exact up to $\varepsilon=10^{-6}$ (or $10^{-12}$, if the probability is close to $10^{-6}$ ). The time column is of the form $\mathrm{xx} / \mathrm{yy}$, where $\mathrm{xx}$ is construction and yy is model check time. A full description of the models is given in [13].

Quasi-Birth-Death Processes [14]. We consider a system consisting of processors and an infinite queue for storing jobs. We compute the probability that, given all processors are busy and there are no jobs in the queue, within $t=10$ time units a state is reached in which all processors are idle and the queue is empty. Results for different rates of incoming jobs $\lambda$ are given below. The depth needed grows linearly with $\lambda$. Thus, the performance of the FSP method suffers from the high cost of repeated transient analysis.

\begin{tabular}{|r|r|c|c|r|r|r|r|r|c|c|}
\hline \multirow{2}{*}{$\lambda$} & \multicolumn{3}{|c|}{ Uniform } & \multicolumn{3}{c|}{ Layered } & \multicolumn{3}{c|}{ FSP } & \multirow{2}{*}{ prob. } \\
\cline { 2 - 10 } & depth & time $(\mathrm{s})$ & $n$ & depth & time $(\mathrm{s})$ & $n$ & depth & time (s) & $n$ & \\
\hline 40 & 609 & $1.1 / 0.2$ & 2,434 & 533 & $1.1 / 0.2$ & 2,130 & 473 & $28.1 / 0.2$ & 1,890 & $4.21 \mathrm{E}-04$ \\
60 & 846 & $1.1 / 0.3$ & 3,382 & 754 & $1.1 / 0.2$ & 3,014 & 694 & $66.0 / 0.2$ & 2,774 & $1.25 \mathrm{E}-04$ \\
80 & 1,077 & $1.1 / 0.3$ & 4,306 & 971 & $1.1 / 0.3$ & 3,882 & 911 & $125.5 / 0.3$ & 3,642 & $5.26 \mathrm{E}-05$ \\
100 & 1,305 & $1.1 / 0.4$ & 5,218 & 1,187 & $1.1 / 0.4$ & 4,746 & 1,127 & $209.4 / 0.4$ & 4,506 & $2.69 \mathrm{E}-05$ \\
\hline
\end{tabular}

Protein Synthesis [15]. We analyze a model of protein synthesis considering the property that, within time $t$ but later than 10 time units, a state is reached, in which 20 or more proteins exist and the synthesizing gene is inactive. Overall, the FSP method is the most efficient here, because a rather low number of layers is relevant. In terms of model construction time, the Uniform method is best here.

\begin{tabular}{|c|c|c|c|c|c|c|c|c|c|c|}
\hline \multirow{2}{*}{$t$} & \multicolumn{3}{|c|}{ Uniform } & \multicolumn{3}{|c|}{ Layered } & \multicolumn{3}{|c|}{ FSP } & \multirow{2}{*}{ prob. } \\
\hline & depth & time $(\mathrm{s})$ & $n$ & depth & time $(\mathrm{s})$ & $n$ & depth & time $(\mathrm{s})$ & $n$ & \\
\hline 100 & 973 & $1.1 / 0.1$ & 1,945 & 756 & $1.1 / 0.1$ & 1,511 & 30 & $1.1 / 0.0$ & 59 & 1. \\
\hline 500 & 3,636 & $1.1 / 6.0$ & 7,271 & 3,308 & $1.2 / 5.0$ & 6,615 & 35 & $1.2 / 0.0$ & 69 & \\
\hline 1000 & 6,830 & $1.1 / 39.3$ & 13,659 & 6,420 & $1.4 / 34.7$ & 12,839 & 36 & $1.4 / 0.0$ & 71 & $9.99 \mathrm{E}-02$ \\
\hline 2000 & 13,103 & $1.1 / 276.9$ & 26,205 & 12,577 & $2.3 / 255.3$ & 25,153 & 37 & $1.7 / 0.0$ & 73 & 2.02E-01 \\
\hline
\end{tabular}

Grid-World Robot [11]. We consider a grid world in which a robot moves from the lower left to the upper right corner sending signals to a station. A janitor moves around and may block the robot for some time. In contrast to [11], the janitor can leave the $N \times N$ field of the robot, which leads to an infinite state space. We check a nested formula of the form mentioned in the introduction, namely whether the robot moves with probability 0.9 to the upper right corner within 100 time units while periodically communicating with the base. We give 
results for different $N$. Using the FSP method is advantageous, as each layer of the model contains quite a large number of states, and FSP only needs to explore a fraction of the layers compared to the other methods. As seen from the "prob." column, for $N=2,3$ the property holds, while, for $N=4$, it does not. The reason for the decreasing probability is that the distance the robot has to travel increases with $n$. Thus the probability decreases that the robot will complete its travel in time.

An implicit representation of the edges of the model used to handle the large number of transitions. However, this increases the runtime in this example.

\begin{tabular}{|c|c|c|c|c|c|c|c|c|c|c|}
\hline \multirow{2}{*}{$N$} & \multicolumn{3}{|c|}{ Uniform } & \multicolumn{3}{|c|}{ Layered } & \multicolumn{3}{|c|}{ FSP } & \multirow{2}{*}{ prob. } \\
\hline & depth & time $(\mathrm{s})$ & $n$ & depth & time $(\mathrm{s})$ & $n$ & depth & time $(\mathrm{s})$ & $n$ & \\
\hline 2 & 905 & $15 / 4,479$ & $9,807 \mathrm{~K}$ & 570 & $6 / 1,667$ & $3885 \mathrm{~K}$ & 107 & $1,417 / 37$ & $135 \mathrm{~K}$ & .000 \\
\hline 3 & 905 & $24 / 8,135$ & $16,308 \mathrm{~K}$ & 571 & $11 / 2,979$ & $6,475 \mathrm{~K}$ & 96 & $1,681 / 56$ & $177 \mathrm{~K}$ & 0.902 \\
\hline 4 & 905 & $33 / 13,323$ & $22,781 \mathrm{~K}$ & 571 & $14 / 5,099$ & $9,034 \mathrm{~K}$ & 98 & $2,754 / 99$ & $253 \mathrm{~K}$ & 0.898 \\
\hline
\end{tabular}

Workstation cluster [16]. We consider the dependability of a fault-tolerant workstation cluster, a model with finite state space. For the model with 512 workstations, we compute the probability that the QoS drops below minimum quality within one time unit, and compare with PRISM. Results are given for the fastest configuration of PRISM (sparse engine), and INFAMY (FSP) respectively.

The state space explored by PRISM has depth 1029 and includes 9.4 million states. Up to $t=20$, INFAMY with FSP is faster than PRISM. However, for larger time bounds, the model construction time dominates, since for each layer the error estimate is recomputed. As observed by Munsky [5], this can be alleviated by adding more than one layer at each step. We consider a variant in which we double the number of layers we add per step, thus computing an error estimate every $1,2,4,8, \ldots$ layers. We call this configuration FSP exponential. It is consistently the fastest method for $t \leq 50$, as shown in the last column of the table.

\begin{tabular}{|r|c|r|r|r|r|r|r|r|}
\hline \multirow{2}{*}{$t$} & PRISM & \multicolumn{3}{|c|}{ FSP } & \multicolumn{2}{|c|}{ FSP exponential } & \multirow{2}{*}{ prob. } \\
\cline { 2 - 7 } & time (s) & depth & \multicolumn{1}{c|}{ time (s) } & \multicolumn{1}{c|}{$n$} & depth & \multicolumn{1}{c|}{ time $(\mathrm{s})$} & \multicolumn{1}{c|}{$n$} & \\
\hline 5.0 & $4.8 / 147.3$ & 37 & $7.1 / 0.4$ & $23 \mathrm{~K}$ & 64 & $3.1 / 1.5$ & $701 \mathrm{~K}$ & $1.21 \mathrm{E}-06$ \\
10.0 & $5.8 / 190.5$ & 52 & $25.0 / 1.4$ & $46 \mathrm{~K}$ & 64 & $4.4 / 2.4$ & $701 \mathrm{~K}$ & $3.79 \mathrm{E}-06$ \\
20.0 & $6.0 / 315.3$ & 80 & $209.1 / 7.8$ & $111 \mathrm{~K}$ & 128 & $26.9 / 20.2$ & $289 \mathrm{~K}$ & $1.01 \mathrm{E}-05$ \\
30.0 & $5.6 / 365.6$ & 104 & $547.5 / 15.1$ & $190 \mathrm{~K}$ & 128 & $38.7 / 28.3$ & $289 \mathrm{~K}$ & $1.68 \mathrm{E}-05$ \\
50.0 & $5.4 / 502.5$ & 147 & $2,610.5 / 46.2$ & $382 \mathrm{~K}$ & 256 & $255.8 / 182.0$ & $1,167 \mathrm{~K}$ & $3.04 \mathrm{E}-05$ \\
\hline
\end{tabular}

Tandem Queueing Network [17]. This model consists of two interconnected queues with capacity $c$. We consider the probability that the first queue becomes full before time 0.23. Larger time bounds are left out, since then this probability is one. The performance results for INFAMY using configuration Layered are better, while for large time bound $(t \geq 1)$ the whole model will be explored thus PRISM will perform better. 


\begin{tabular}{|r|r|r|r|r|r|r|r|}
\hline \multirow{2}{*}{$c$} & \multicolumn{3}{|c|}{ PRISM } & \multicolumn{3}{|c|}{ Layered } & \multirow{2}{*}{ prob. } \\
\cline { 2 - 7 } & depth & \multicolumn{1}{|c|}{ time } & \multicolumn{1}{|c|}{$n$} & depth & \multicolumn{1}{c|}{$n$} & \\
\hline 511 & 1,535 & $3.7 / 49.7$ & 523,776 & 632 & $6.8 / 7.5$ & 235,339 & $3.13 \mathrm{E}-02$ \\
1023 & 3,071 & $13.7 / 380.3$ & $2,096,128$ & 1,167 & $3.6 / 49.6$ & 714,306 & $4.24 \mathrm{E}-03$ \\
2047 & 6,143 & $69.3 / 3,068.3$ & $8,386,560$ & 2,198 & $10.0 / 297.8$ & $2,449,798$ & $9.87 \mathrm{E}-05$ \\
4095 & 12,287 & $560.9 / 31,386.5$ & $33,550,336$ & 4,209 & $27.4 / 2,889.8$ & $8,899,113$ & $7.06 \mathrm{E}-08$ \\
\hline
\end{tabular}

\section{Conclusion and Future Work}

INFAMY enables model checking of infinite models and, for certain finite models, is competitive with the leading model checker PRISM in time and memory usage. As observed in our experiments, the appropriate truncation method strongly depends on the model and property under consideration. Therefore, INFAMY allows the user to select the method that fits best. We plan to implement heuristics that choose the estimation method automatically. Further, the approach implemented in INFAMY is applicable to richer languages than PRISM, e.g., languages that support dynamic process creation, as in the stochastic $\pi$-calculus.

\section{References}

1. Aziz, A., Sanwal, K., Singhal, V., Brayton, R.K.: Model-Checking ContinuousTime Markov Chains. ACM Trans. Comput. Log 1, 162-170 (2000)

2. Fox, B.L., Glynn, P.W.: Computing Poisson Probabilities. Commun. ACM 31, 440-445 (1988)

3. Grassmann, W.K.: Finding Transient Solutions in Markovian Event Systems Through Randomization. In: NSMC 1991, pp. 357-371 (1991)

4. van Moorsel, A.P.A., Sanders, W.H.: Adaptive Uniformization. Communications in Statistics - Stochastic Models 10, 619-647 (1994)

5. Munsky, B., Khammash, M.: The Finite State Projection Algorithm for the Solution of the Chemical Master Equation. Journal of Chemical Physics 124 (2006)

6. Zhang, L., Hermanns, H., Hahn, E.M., Wachter, B.: Time-Bounded Model Checking of Infinite-State Continuous-Time Markov Chains. In: ACSD, pp. 98-107 (2008)

7. Holzmann, G.J.: The Model Checker SPIN. IEEE Trans. Software Eng. 23, 279-295 (1997)

8. Baier, C., Haverkort, B.R., Hermanns, H., Katoen, J.P.: Model checking meets performance evaluation. SIGMETRICS Performance Evaluation Review 32, 10-15 (2005)

9. Hinton, A., Kwiatkowska, M.Z., Norman, G., Parker, D.: PRISM: A Tool for Automatic Verification of Probabilistic Systems. In: Hermanns, H., Palsberg, J. (eds.) TACAS 2006. LNCS, vol. 3920, pp. 441-444. Springer, Heidelberg (2006)

10. Hérault, T., Lassaigne, R., Magniette, F., Peyronnet, S.: Approximate Probabilistic Model Checking. In: Steffen, B., Levi, G. (eds.) VMCAI 2004. LNCS, vol. 2937, pp. 73-84. Springer, Heidelberg (2004)

11. Younes, H.L.S.: Ymer: A statistical model checker. In: Etessami, K., Rajamani, S.K. (eds.) CAV 2005. LNCS, vol. 3576, pp. 429-433. Springer, Heidelberg (2005)

12. Zapreev, I.S.: Model Checking Markov Chains: Techniques and Tools. Ph.D thesis, University of Twente, Enschede, The Netherlands (2008) 
13. Hahn, E.M., Hermanns, H., Wachter, B., Zhang, L.: Time-Bounded Model Checking of Infinite-State Continuous-Time Markov Chains. Technical Report No. 47, SFB/TR 14 AVACS (2009); To appear in Fundamenta Informaticae

14. Katoen, J.P., Klink, D., Leucker, M., Wolf, V.: Three-Valued Abstraction for Continuous-Time Markov Chains. In: Damm, W., Hermanns, H. (eds.) CAV 2007. LNCS, vol. 4590, pp. 311-324. Springer, Heidelberg (2007)

15. Gross, P.J.E., Peccoud, J.: Quantitative modeling of stochastic systems in molecular biology by using stochastic Petri nets. Proc. Natl. Acad. Sci. USA 95, 6750-6755 (1998)

16. Haverkort, B.R., Hermanns, H., Katoen, J.P.: On the Use of Model Checking Techniques for Dependability Evaluation. In: SRDS, pp. 228-237 (2000)

17. Hermanns, H., Meyer-Kayser, J., Siegle, M.: Multi terminal binary decision diagrams to represent and analyse continuous time Markov chains. In: Plateau, B., Stewart, W., Silva, M. (eds.) NSMC, pp. 188-207 (1999) 